\title{
DEALING WITH BONE METASTASES FROM BREAST CANCER - A PATHOLOGICAL AND CLINICAL OVERVIEW
}

doi: 10.2478/rojost-2018-0036

\author{
O. Munteanu $u^{1,2}$, A. Dumitru ${ }^{2,3}$, O. Bodean ${ }^{1}$, L. Arsene ${ }^{1}$, D. Voicu ${ }^{1}$, E. Bratilă ${ }^{2,4}$, C. Mehedințu $u^{2,5}$, M. Sajin ${ }^{2,3}$, \\ M.M. Cîrstoiu ${ }^{1,2}$ \\ ${ }^{1}$ Obstetrics and Gynecology Department, University Emergency Hospital, Bucharest, Romania \\ 2 "Carol Davila" University of Medicine and Pharmacy, Bucharest, Romania \\ ${ }^{3}$ Department of Morphopathology, University Emergency Hospital, Bucharest, Romania \\ 4Obstetrics and Gynecology Department, "Panait Sârbu" Emergency Hospital, Bucharest, Romania \\ 5Obstetrics and Gynecology Department, "Nicolae Malaxa" Emergency Hospital, Bucharest, Romania
}

From breast malignant tumors, bone is the most frequent site of metastasis. Bone metastases from breast cancer are correlated with pathological fractures, spinal cord compression and other skeletal-related events as well as bone pain and hypercalcemia. These lead to impaired mobility, decreased quality of life, and overall decrease in survival. Clarification of mechanisms regulating bone metastasis has advanced greatly in the last years and this has translated into plentiful unused therapeutic options. Greater understanding of the pathophysiology of bone metastases has led to the detection and clinical efficiency of bone-targeted agents. This review summarizes the key evidence for current clinical practice and future directions.

Keywords: metastases, bone, breast cancer, pathology 